\title{
Numerical simulation for a class of predator-prey system with homogeneous Neumann boundary condition based on a sinc function interpolation method
}

\author{
Dandan Dai ${ }^{1,2}$, Ximing $\mathrm{Lv}^{3}$ and Yulan Wang ${ }^{1 *}$ (D)
}

"Correspondence: wylnei@163.com 'Department of Mathematics, Inner Mongolia University of Technology, Hohhot, P.R. China

Full list of author information is available at the end of the article

\begin{abstract}
For the nonlinear predator-prey system (PPS), although a variety of numerical methods have been proposed, such as the difference method, the finite element method, and so on, but the efficient numerical method has always been the direction that scholars strive to pursue. Based on this question, a sinc function interpolation method is proposed for a class of PPS. Numerical simulations of a class of PPS with complex dynamical behaviors are performed. Time series plots and phase diagrams of a class of PPS without self-diffusion are shown. The pattern is obtained by setting up different initial conditions and the parameters in the system according to Turing bifurcation condition. The numerical simulation results have a good agreement with theoretical results. Simulation results show the effectiveness of the method.
\end{abstract}

Keywords: Reaction-diffusion system; Spectral interpolation method; Complex dynamical behavior; Numerical simulation

\section{Introduction}

The PPS is a basic ecological system that exists widely in nature and is an essential component of ecosystems such as oceans, lakes, wetlands, forests, and grasslands. The predatory process plays an important role in promoting life evolution, maintaining ecological balance, and maintaining biodiversity. Therefore, research on PPS is crucial to the exploration of the fundamental nature of ecosystems. In [1, 2], a PPS with BeddingtonDeAngelis-type functional response is proposed and analyzed. In [3], a PPS with general Holling type functional response is given. In [4], a modified Leslie-Gower-type PPS with Holling's type II functional response is studied. In [5], Paul and Ghosh gave preypredator-generalist predator system of the following form:

$$
\left\{\begin{array}{l}
\frac{d x}{d t}=r x\left(1-\frac{x}{k}\right)-\alpha_{0} x y-\beta_{0} x z, \\
\frac{d y}{d t}=\alpha_{1} x y-\gamma_{0} y z-m_{1} y, \\
\frac{d z}{d t}=\beta_{1} x z+\gamma_{1} y z-m_{2} z,
\end{array}\right.
$$

(c) The Author(s) 2020. This article is licensed under a Creative Commons Attribution 4.0 International License, which permits use sharing, adaptation, distribution and reproduction in any medium or format, as long as you give appropriate credit to the original author(s) and the source, provide a link to the Creative Commons licence, and indicate if changes were made. The images or other third party material in this article are included in the article's Creative Commons licence, unless indicated otherwise in a credit line to the material. If material is not included in the article's Creative Commons licence and your intended use is not permitted by statutory regulation or exceeds the permitted use, you will need to obtain permission directly from the copyright holder. To view a copy of this licence, visit http://creativecommons.org/licenses/by/4.0/. 
with the initial conditions

$$
x(0)=c_{1}, y(0)=c_{2}, z(0)=c_{3},
$$

where $x, y$, and $z$ are respectively the prey biomass, predator biomass, and top predator biomass at any time $\mathrm{t}, \mathrm{r}$ and $\mathrm{k}$ are respectively the intrinsic growth rate and the environmental carrying capacity of prey species. $\alpha_{0}, \beta_{0}, \gamma_{0}, \alpha_{1}, \beta_{1}, \gamma_{1}, m_{1}, m_{2}$ are all parameters.

When studying the spatial distribution structure and maintaining biodiversity $[6,7]$ of predators and prey populations, the reaction diffusion system [8-13] can more accurately describe the interaction between predators and preys. In the PPS, spatial diffusion is reflected in the predator's efforts to catch up with the prey, and the prey's efforts to escape the predator's pursuit. In [5], if diffusion behavior of predator-prey biomass is considered, the following system can be obtained [14]:

$$
\left\{\begin{array}{l}
\frac{\partial \eta}{\partial t}=d_{1} \Delta \eta+r \eta\left(1-\frac{\eta}{k}\right)-\alpha_{0} \eta u-\beta_{0} \eta v \\
\frac{\partial u}{\partial t}=d_{2} \Delta u+\alpha_{1} \eta u-\gamma_{0} u v-m_{1} u \\
\frac{\partial v}{\partial t}=d_{3} \Delta v+\beta_{1} \eta v+\gamma_{1} u v-m_{2} v
\end{array}\right.
$$

where $\eta=\eta(x, y, t), u=u(x, y, t)$, and $v=v(x, y, t)$ are respectively the prey biomass, predator biomass, and top predator biomass at any time $t, r$ and $k$ are respectively the intrinsic growth rate and environmental carrying capacity of the prey species. $\alpha_{0}, \beta_{0}$ are respectively the predation rate of the predator and top predator on prey species. $\alpha_{1}$ and $\beta_{1}$ are respectively measure of the conversion rate of prey species to its predator species and $\gamma_{1}$ is the conversion rate of predator species to the top predator species. $\gamma_{0}$ is the predation rate of top predator on predator species. $m_{1}$ and $m_{2}$ are respectively the natural death rate of the predator and top predator; and $d_{1}, d_{2}$, and $d_{3}$ are positive diffusion coefficients, $\Delta=\frac{\partial^{2}}{\partial x^{2}}+\frac{\partial^{2}}{\partial y^{2}} .(x, y) \in \Omega=[a, b] \times[c, d]$, the smooth boundary is $\partial \Omega$, homogeneous Neumann boundary condition, namely $\left.\frac{\partial u}{\partial n}\right|_{\partial \Omega}=\left.\frac{\partial v}{\partial n}\right|_{\partial \Omega}=0$.

For the nonlinear PPS, a variety of numerical methods have been proposed, such as finite difference method [15], B-spline method [16], finite element method [17, 18], spectral method [19-21], the perturbation method and variational iteration method (VIM) [22, 23], barycentric interpolation collocation method (BICM) [24-27], reproducing kernel method (RKM) [28-32], etc. Nevertheless, the efficient numerical method has always been the direction that scholars strived to pursue. Based on this question, a sinc function interpolation method is proposed for a class of PPS.

\section{Bifurcation analysis of system}

PPS (2) has at most five equilibrium points as follows:

(i) the trivial equilibrium $p_{0}(0,0,0)$;

(ii) the predator-free equilibrium $P_{1}(k, 0,0)$;

(iii) the top predator-free equilibrium $P_{2}\left(\frac{m_{1}}{\alpha_{1}}, r \frac{k \alpha_{1}-m_{1}}{k \alpha_{0} \alpha_{1}}, 0\right)$;

(iv) the predator-free equilibrium $P_{3}\left(\frac{m_{2}}{\beta_{1}}, 0, r \frac{k \beta_{1}-m_{2}}{k \beta_{0} \beta_{1}}\right)$;

(v) the coexistence equilibrium $p_{4}\left(\frac{k m_{1} \beta_{0}+r \gamma_{0} \gamma_{1}-m_{2} \alpha_{0} \gamma_{0}}{\vartheta}, \frac{r m_{2} \gamma_{0}+k\left(m_{2} \alpha_{1} \beta_{0}-m_{1} \beta_{0} \beta_{1}-r \beta_{1} \gamma_{0}\right)}{\vartheta}, \frac{k\left(r \alpha_{1} \gamma_{1}+m_{1} \alpha_{0} \beta_{1}-m_{2} \alpha_{0} \alpha_{1}\right)-r m_{1} \gamma_{1}}{\vartheta}\right)$, where $\vartheta=r \gamma_{0} \gamma_{1}+k\left(\alpha_{1} \beta_{0} \gamma_{1}-\alpha_{0} \beta_{1} \gamma_{0}\right)$. 
The following equation at arbitrary equilibrium point $(\eta, u, v)$ is given [5]:

$$
\left\{\begin{array}{l}
r \eta\left(1-\frac{\eta}{k}\right)-\alpha_{0} \eta u-\beta_{0} \eta v=0 \\
\alpha_{1} \eta u-\gamma_{0} u v-m_{1} u=0 \\
\beta_{1} \eta v+\gamma_{1} u v-m_{2} v=0
\end{array}\right.
$$

From the biological point of view we are only interested in the stability behavior of the positive equilibrium point. Obviously, the trivial equilibrium $P_{0}$ and the predator-free equilibrium $P_{1}$ always exist. The top predator-free equilibrium $P_{2}$ exists if $k \alpha_{1}>m_{1}$, and the coexistence equilibrium $P_{4}$ exists if $r \gamma_{0} \gamma_{1}+k \alpha_{1} \beta_{0} \gamma_{1}>k \alpha_{0} \beta_{1} \gamma_{0}$. It is also to be noted that the existence of the equilibrium $P_{4}$ ensures the existence of the remaining equilibria.

The Jacobian matrix of nondiffusive system (2) at arbitrary equilibrium point $(\eta, u, v)$ is given as follows:

$$
A_{0}=\left[\begin{array}{ccc}
r-\frac{2 r \eta}{k}-\alpha_{0} u-\beta_{0} v & -\alpha_{0} \eta & -\beta_{0} \eta \\
\alpha_{1} u & \alpha_{1} \eta-\gamma_{0} v-m_{1} & -\gamma_{0} u \\
\beta_{1} v & \gamma_{1} v & \beta_{1} \eta+\gamma_{1} u-m_{2}
\end{array}\right] .
$$

The Jacobian matrix $A_{\lambda}$ of system (2) is

$$
A_{\lambda}=\left[\begin{array}{ccc}
r-\frac{2 r \eta}{k}-\alpha_{0} u-\beta_{0} v & -\alpha_{0} \eta & -\beta_{0} \eta \\
\alpha_{1} u & \alpha_{1} \eta-\gamma_{0} v-m_{1} & -\gamma_{0} u \\
\beta_{1} v & \gamma_{1} v & \beta_{1} \eta+\gamma_{1} u-m_{2}
\end{array}\right]-\lambda^{2}\left[\begin{array}{ccc}
d_{1} & 0 & 0 \\
0 & d_{2} & 0 \\
0 & 0 & d_{3}
\end{array}\right] .
$$

Turing bifurcation occurs when the equilibrium state is stable in absence of nondiffusion, but it becomes unstable in presence of cross-diffusion. Thus, if there exists $\lambda$, the the equilibrium state becomes an unstable point of the cross-diffusion system (2), and if the real part of eigenvalues $A_{k}$ is positive, then diffusion system (2) is unstable.

\section{Description of the sinc function interpolation method}

To solve system (2), we consider a regular region $\Omega=[0,2 \pi] \times[0,2 \pi]$, the interval $[0,2 \pi]$ is divided into $N$ different nodes. $h=\frac{2 \pi}{N}, x_{j}=j h, y_{j}=j h, j=1,2 \ldots, N$. Sinc functions are used in different areas of physics and mathematics. A periodic sinc function

$$
S_{N}(x)=\frac{\sin (\pi x / h)}{(2 \pi / h) \tan (x / 2)},
$$

where $h=\frac{2 \pi}{N}, S_{N}$ is the interpolation function of periodic $\delta$ function. It can be proved that $\left.S_{N}(x)\right|_{x \rightarrow 0}=1 . S_{N}\left(x_{i}-x_{j}\right)$ is an $N$ order unit matrix, respectively.

Using periodic sinc function (6), for given $h>0$, we define the following interpolation space:

$$
\operatorname{Span}\left\{S_{N}(x-j h), j=1,2, \ldots, N\right\} .
$$

Let $I_{N}$ be the interpolation operator such that for functions $\eta(x, y, t), u(x, y, t)$, and $v(x, y, t)$ defined on $[0,2 \pi]$ with homogeneous Neumann boundary condition, the interpolation functions $I_{N} \eta(x, y, t), I_{N} u(x, y, t)$, and $I_{N} v(x, y, t)$ of sequence $\eta_{i, j}=\eta\left(x_{i}, y_{j}, t\right), u_{i, j}=u\left(x_{i}, y_{j}, t\right)$, 
$v_{i, j}=v\left(x_{i}, y_{j}, t\right)$ can be written as follows [33]:

$$
\begin{aligned}
& \eta(x, y, t) \sim I_{N} \eta(x, y, t)=\sum_{i=1}^{N} \sum_{j=1}^{N} S_{N}\left(x-x_{i}\right) S_{N}\left(y-y_{i}\right) \eta\left(x_{i}, y_{j}, t\right), \\
& u(x, y, t) \sim I_{N} u(x, y, t)=\sum_{i=1}^{N} \sum_{j=1}^{N} S_{N}\left(x-x_{i}\right) S_{N}\left(y-y_{i}\right) u\left(x_{i}, y_{j}, t\right), \\
& v(x, y, t) \sim I_{N} v(x, y, t)=\sum_{i=1}^{N} \sum_{j=1}^{N} S_{N}\left(x-x_{i}\right) S_{N}\left(y-y_{i}\right) v\left(x_{i}, y_{j}, t\right) .
\end{aligned}
$$

At collocation nodes $\left(x_{p}, y_{q}\right)$, the following relations hold:

$$
\begin{aligned}
& \eta\left(x_{p}, y_{q}, t\right)=I_{N} \eta\left(x_{p}, y_{q}, t\right)=\sum_{i=1}^{N} \sum_{j=1}^{N} S_{N}\left(x_{p}-x_{i}\right) S_{N}\left(y_{q}-y_{i}\right) \eta\left(x_{i}, y_{j}, t\right) \\
& u\left(x_{p}, y_{q}, t\right)=I_{N} u\left(x_{p}, y_{q}, t\right)=\sum_{i=1}^{N} \sum_{j=1}^{N} S_{N}\left(x_{p}-x_{i}\right) S_{N}\left(y_{q}-y_{i}\right) u\left(x_{i}, y_{j}, t\right) \\
& v\left(x_{p}, y_{q}, t\right)=I_{N} v\left(x_{p}, y_{q}, t\right)=\sum_{i=1}^{N} \sum_{j=1}^{N} S_{N}\left(x_{p}-x_{i}\right) S_{N}\left(y_{q}-y_{i}\right) v\left(x_{i}, y_{j}, t\right), \\
& \eta^{(l, k)}\left(x_{p}, y_{q}, t\right) \sim I_{N} \eta^{(l, k)}\left(x_{p}, y_{q}, t\right)=\frac{\partial^{l+k} \eta\left(x_{p}, y_{q}, t\right)}{\partial x^{l} \partial y^{k}} \\
& =\sum_{i=1}^{N} \sum_{j=1}^{N} S_{N}^{(l)}\left(x_{p}-x_{i}\right) S_{N}^{(k)}\left(y_{q}-y_{i}\right) \eta\left(x_{i}, y_{j}, t\right) \\
& u^{(l, k)}\left(x_{p}, y_{q}, t\right) \sim I_{N} u^{(l, k)}\left(x_{p}, y_{q}, t\right)=\frac{\partial^{l+k} u\left(x_{p}, y_{q}, t\right)}{\partial x^{l} \partial y^{k}} \\
& =\sum_{i=1}^{N} \sum_{j=1}^{N} S_{N}^{(l)}\left(x_{p}-x_{i}\right) S_{N}^{(k)}\left(y_{q}-y_{i}\right) u\left(x_{i}, y_{j}, t\right), \\
& v^{(l, k)}\left(x_{p}, y_{q}, t\right) \sim I_{N} v^{(l, k)}\left(x_{p}, y_{q}, t\right)=\frac{\partial^{l+k} v\left(x_{p}, y_{q}, t\right)}{\partial x^{l} \partial y^{k}} \\
& =\sum_{i=1}^{N} \sum_{j=1}^{N} S_{N}^{(l)}\left(x_{p}-x_{i}\right) S_{N}^{(k)}\left(y_{q}-y_{i}\right) v\left(x_{i}, y_{j}, t\right) .
\end{aligned}
$$

Noting

$$
\begin{aligned}
& \boldsymbol{\eta}=\left[\eta_{11}, \eta_{21}, \ldots, \eta_{N 1}, \eta_{12}, \eta_{22}, \ldots, \eta_{N 2}, \eta_{1 N}, \ldots, \eta_{N N}\right]^{T}, \\
& \mathbf{u}=\left[u_{11}, u_{21}, \ldots, u_{N 1}, u_{12}, u_{22}, \ldots, u_{N 2}, u_{1 N}, \ldots, u_{N N}\right]^{T}, \\
& \mathbf{v}=\left[v_{11}, v_{21}, \ldots, v_{N 1}, v_{12}, v_{22}, \ldots, v_{N 2}, v_{1 N}, \ldots, v_{N N}\right]^{T} .
\end{aligned}
$$

Therefore, formula (8) can be written as the following matrix form:

$$
\begin{aligned}
& \boldsymbol{\eta}=D^{(0,0)} \boldsymbol{\eta}, \quad \mathbf{u}=D^{(0,0)} \mathbf{u}, \quad \mathbf{v}=D^{(0,0)} \mathbf{v}, \\
& \boldsymbol{\eta}^{(l, k)}=D_{N}^{(l, k)} \boldsymbol{\eta}, \quad \mathbf{u}^{(l, k)}=D_{N}^{(l, k)} \mathbf{u}, \quad \mathbf{v}^{(l, k)}=D_{N}^{(l, k)} \mathbf{v},
\end{aligned}
$$


where $D_{N}^{(l, k)}=D_{N}^{(l)} \otimes D_{N}^{(k)}$ is the Kronecker product of matrix $D_{N}^{(l)}$ and $D_{N}^{(k)}$, and $D^{(0,0)}=$ $I_{N} \otimes I_{N}, D_{N}^{(0)}=I_{N}, I_{N}$ is an $N$ order unit matrix, respectively.

Employing Eqs. (7), (9), and (10), the discrete form of Eq. (2) can be written as follows:

$$
\frac{\partial}{\partial t}\left[\begin{array}{l}
\eta \\
\mathbf{u} \\
\mathbf{v}
\end{array}\right]=\left[\begin{array}{ccc}
d_{1} D+m_{0} E & 0 & 0 \\
0 & d_{2} D-m_{1} E & 0 \\
0 & 0 & d_{3} D+m_{2} E
\end{array}\right]\left[\begin{array}{l}
\eta \\
\mathbf{u} \\
\mathbf{v}
\end{array}\right]=\left[\begin{array}{l}
f_{1}(\boldsymbol{\eta}, \mathbf{u}, \mathbf{v}) \\
f_{2}(\boldsymbol{\eta}, \mathbf{u}, \mathbf{v}) \\
f_{3}(\boldsymbol{\eta}, \mathbf{u}, \mathbf{v})
\end{array}\right] .
$$

Here,

$$
\begin{aligned}
& {[\boldsymbol{\eta}, \mathbf{u}, \mathbf{v}]=\left[\eta_{11}, \ldots, \eta_{N 1}, \eta_{12}, \ldots, \eta_{N N}, u_{11}, \ldots, u_{N 1}, u_{12}, \ldots, u_{N 2}, u_{1 N}, \ldots, u_{N N}\right.} \\
& \left.v_{11}, \ldots, v_{N 1}, v_{12}, \ldots, v_{N 2}, v_{1 N}, \ldots, v_{N N}\right] \\
& D=D_{N}^{(2,0)}+D_{N}^{(0,2)}, \quad E=D^{(0,0)}, \\
& {\left[f_{1}(\boldsymbol{\eta}, \mathbf{u}, \mathbf{v}), f_{2}(\boldsymbol{\eta}, \mathbf{u}, \mathbf{v}), f_{3}(\boldsymbol{\eta}, \mathbf{u}, \mathbf{v})\right]} \\
& =\left[f_{1}\left(\eta_{11}, u_{11}, v_{11}\right), \ldots, f_{1}\left(\eta_{N N}, u_{N N}, v_{N N}\right)\right. \text {, } \\
& \left.f_{2}\left(\eta_{11}, u_{11}, v_{11}\right), \ldots, f_{2}\left(\eta_{N N}, u_{N N}, v_{N N}\right), f_{3}\left(\eta_{11}, u_{11}, v_{11}\right), \ldots, f_{3}\left(\eta_{N N}, u_{N N}, v_{N N}\right)\right] \text {, } \\
& f_{1}(\eta, u, v)=-m_{0} \frac{\eta}{k}-\alpha_{0} \eta u-\beta_{0} \eta v, \quad f_{2}(\eta, u, v)=\alpha_{1} \eta u-\gamma_{0} u v, \\
& f_{3}(\eta, u, v)=\beta_{1} \eta v+\gamma_{1} u v .
\end{aligned}
$$

Using ode45 in MATLAB to solve Eq. (11) with different initial conditions, we can get the numerical solution of system (2).

\section{Numerical experiments}

In this section, we give some numerical illustrations for better explanation of the above analytical results using different initial conditions and parameters.

Experiment 1 We consider model (1). Taking the parameters $c_{1}=c_{2}=c_{3}=1, d_{1}=d_{2}=$ $d_{3}=0, r=0.1, \alpha_{0}=0.6, \alpha_{1}=0.3, \beta_{0}=0.3, \beta_{1}=\gamma_{0}=0.1, \gamma_{1}=0.08, m_{1}=0.15, m_{2}=0.2$, the prey and the predator survive in the long-run Fig. $1(a)$ and $(b)$. It is also noticed that the top predator always remains at zero level even for large value of $k(=200)$ (see Fig. $1(b)$ ) Taking the parameters $c_{1}=c_{2}=c_{3}=1, d_{1}=d_{2}=d_{3}=0, r=0.1, \alpha_{0}=0.6, \alpha_{1}=0.3, \beta_{0}=0.3$, $\beta_{1}=\gamma_{0}=0.1, \gamma_{1}=0.1, m_{1}=0.08, m_{2}=0.1, k=50$, the top predator does not remain at zero level (see Fig. $1(c)$ ).

Taking the parameters $\beta_{0}=\beta_{1}=0, c_{1}=1, c_{2}=1, c_{3}=1$, and using the present method, time series plots for Experiment 1 with different parameters are given in Fig. 2. Phase

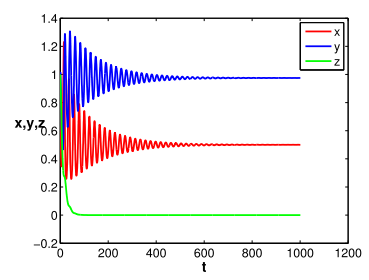

(a)

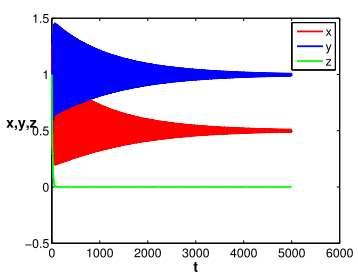

(b)

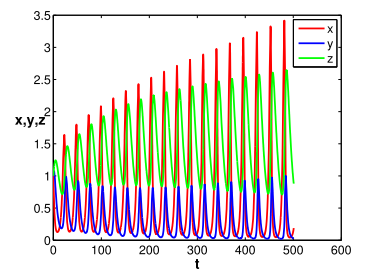

(c)

Figure 1 Time series plots for Experiment 1 with different parameters, for parameters see Table 2 


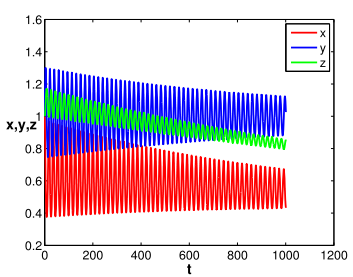

(a)

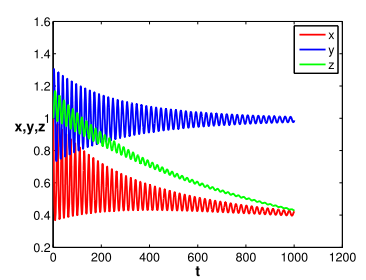

(b)

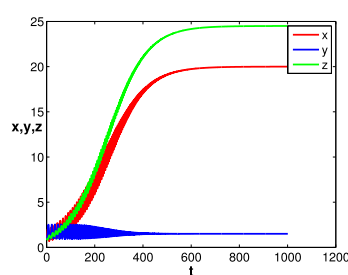

(c)

Figure 2 Time series plots for Experiment 1 with different parameters, for parameters see Table 2

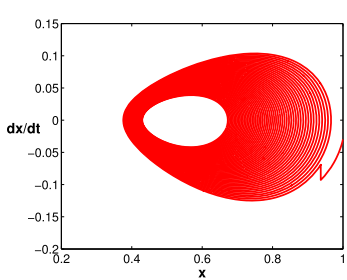

Phase diagram of $x$

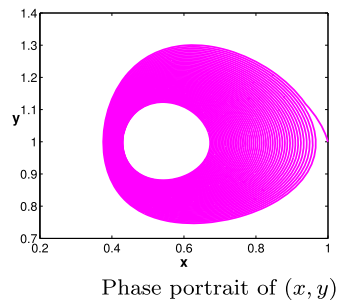

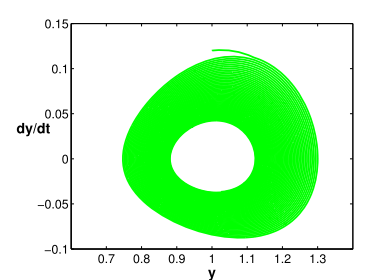

Phase diagram of $y$

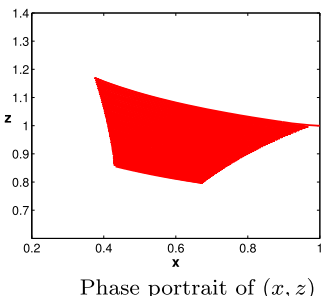

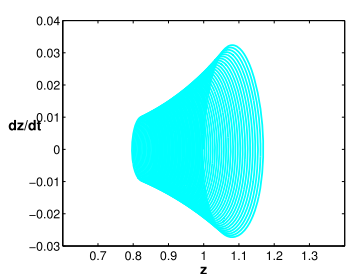

Phase diagram of $z$

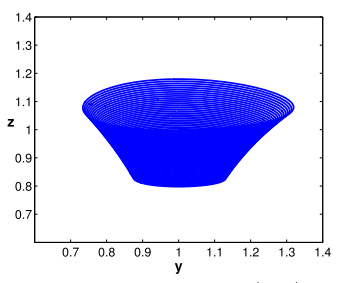

Phase portrait of $(y, z)$

Figure 3 Phase diagram of Experiment 1 with the value of the parameter, for parameters see Table 2
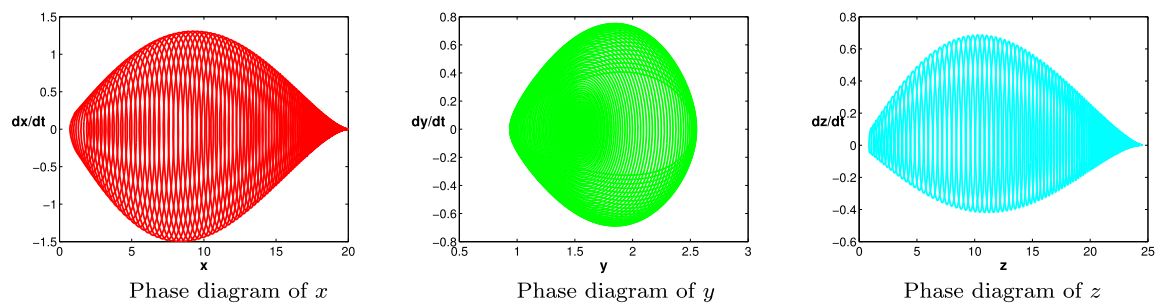

Figure 4 Phase diagram of Experiment 1 with the value of the parameter, for parameters see Table 2

diagram of Experiment 1 with the value of the parameter is shown in Figs. 3-4. Figure 2 shows that the coexistence equilibrium $P_{4}$ exists.

Experiment 2 We consider model (2) with different initial conditions and the parameters $r=0.1, \alpha_{0}=0.6, \alpha_{1}=0.3, \beta_{0}=0.3, \beta_{1}=0.1, \gamma_{0}=0.1, k=1, d_{1}=1, d_{2}=1, d_{3}=1, m_{1}=0.3$, $m_{2}=0.5, \gamma_{1}=1$. Numerical solution and pattern of Experiment 2 are showed in Figs. 5-7.

Experiment 3 We consider model (2) with the parameters $r=0.8, \alpha_{0}=0.4, \alpha_{1}=0.4, \beta_{0}=$ $0.5, \beta_{1}=0.4, \gamma_{0}=0.2, \gamma_{1}=0.5, m_{1}=0.1, m_{2}=0.2, k=2.4, d_{1}=d_{3}=0.1, d_{2}=0.3$. Numerical solution and pattern of Experiment 3 are showed in Figs. 8-11. Tables 1-3 show different parameters and initial conditions in Figs. 1-11. 


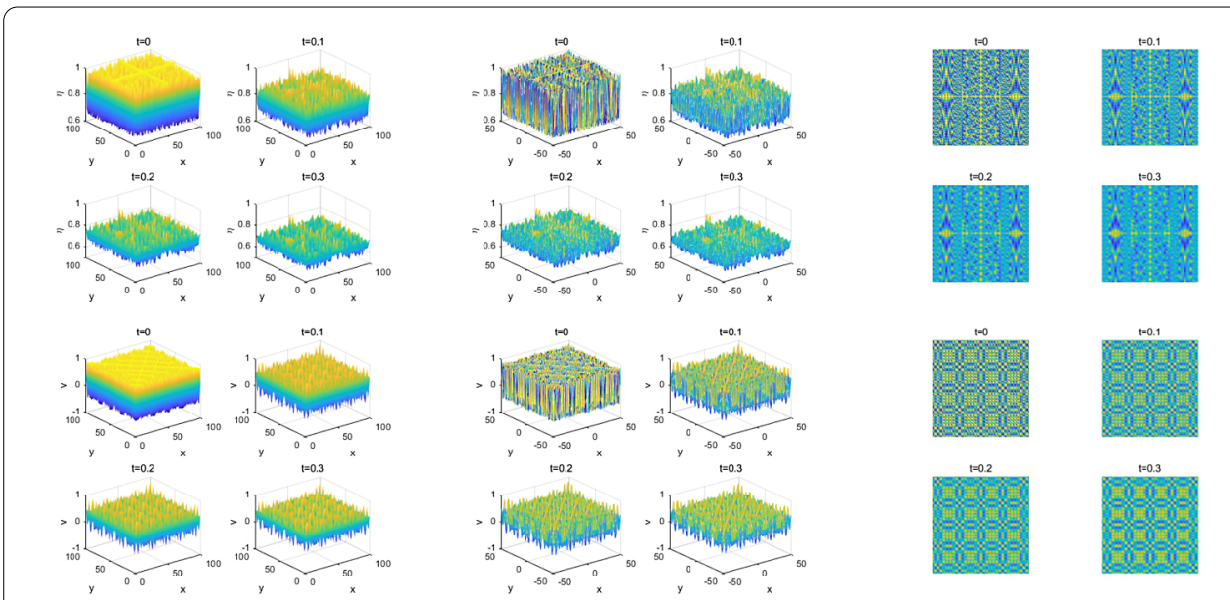

Figure 5 Numerical solution and pattern of Experiment 2, for initial conditions see Table 3

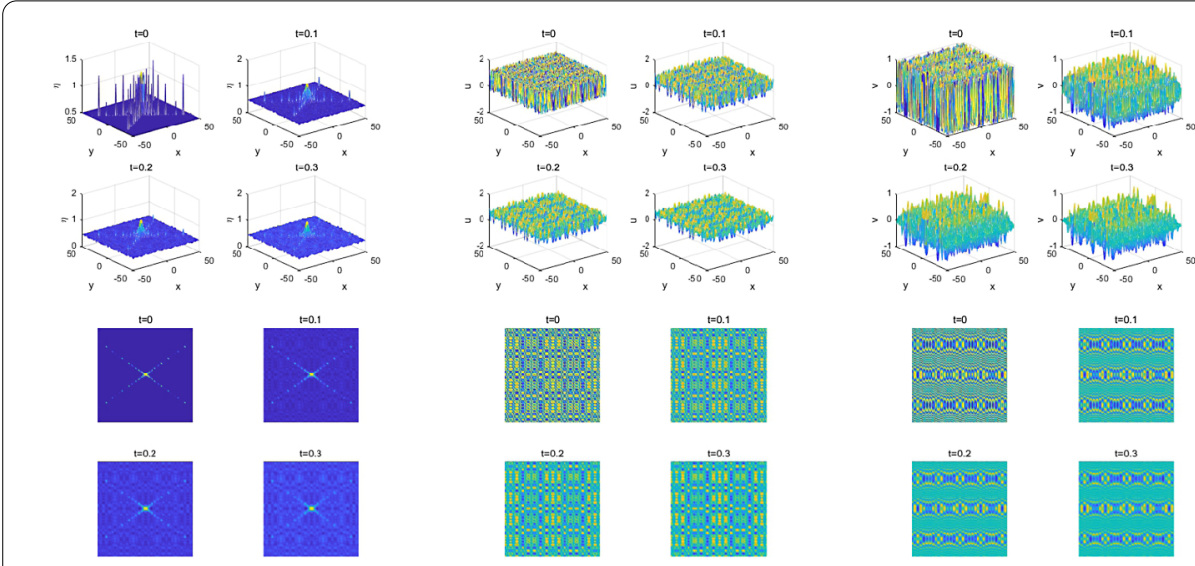

Figure 6 Numerical solution and pattern of Experiment 2, for initial conditions see Table 3

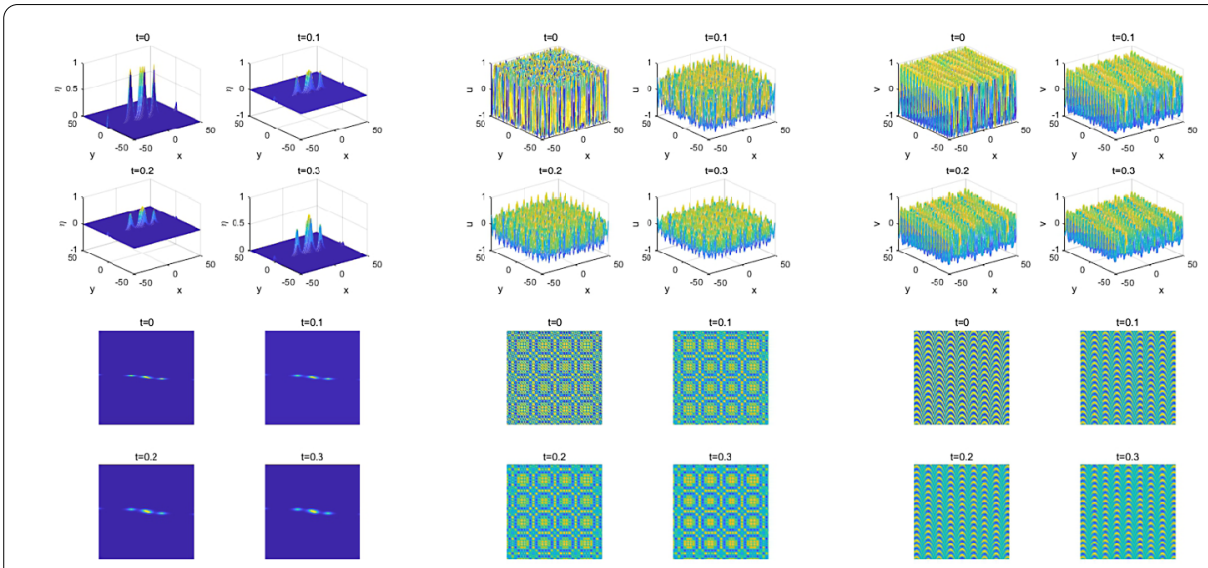

Figure 7 Numerical solution and pattern of Experiment 2, for initial conditions see Table 3 


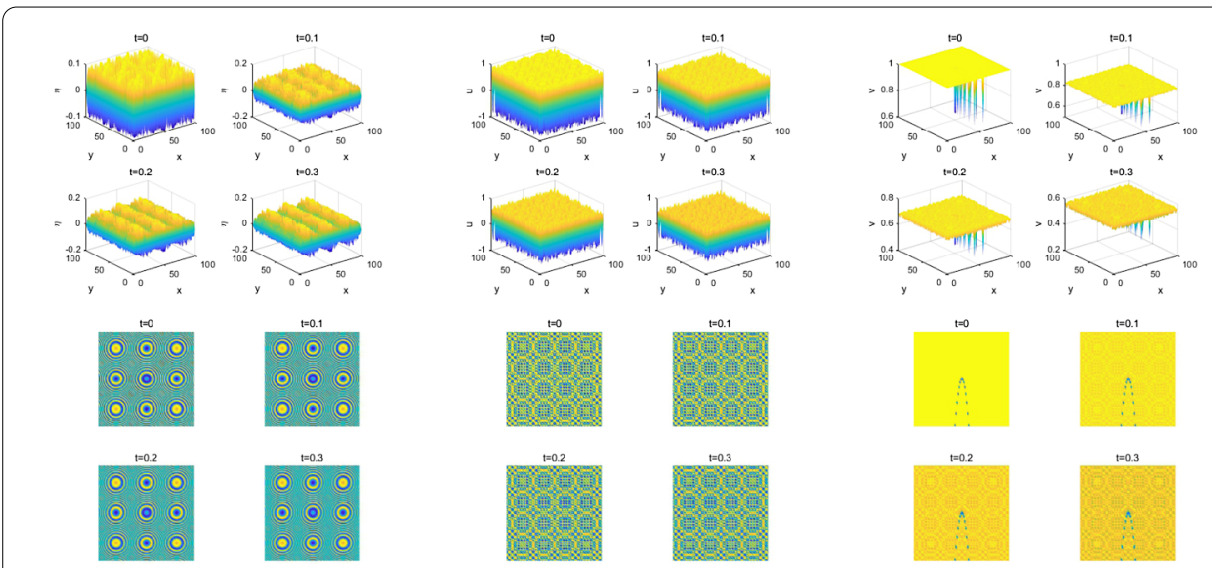

Figure 8 Numerical solution and pattern of Experiment 3, for initial conditions see Table 3

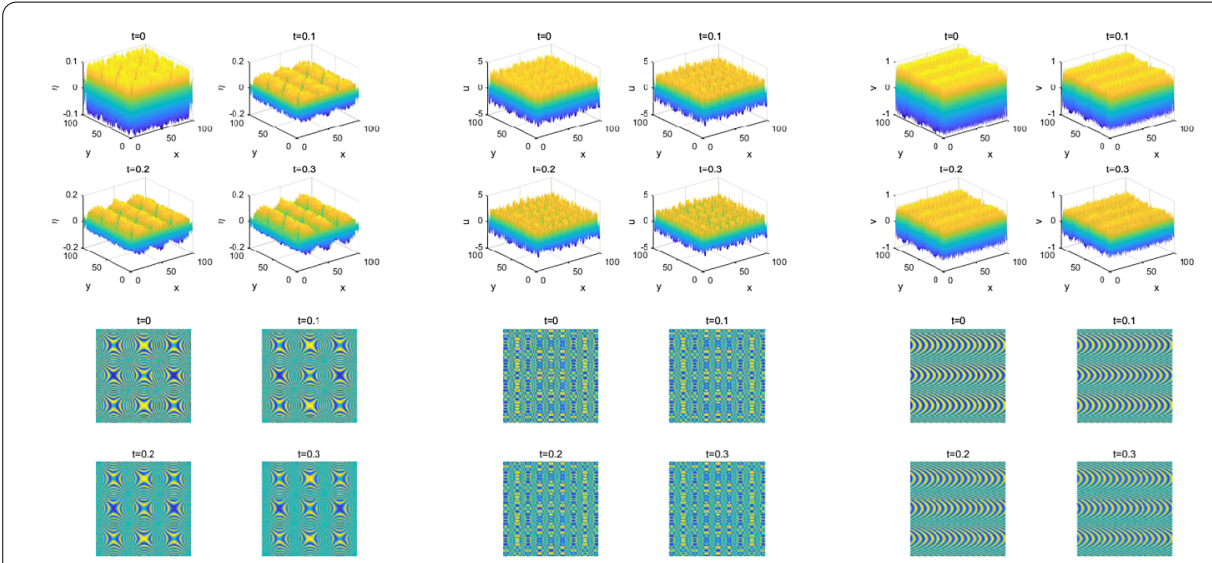

Figure 9 Numerical solution and pattern of Experiment 3, for initial conditions see Table 3

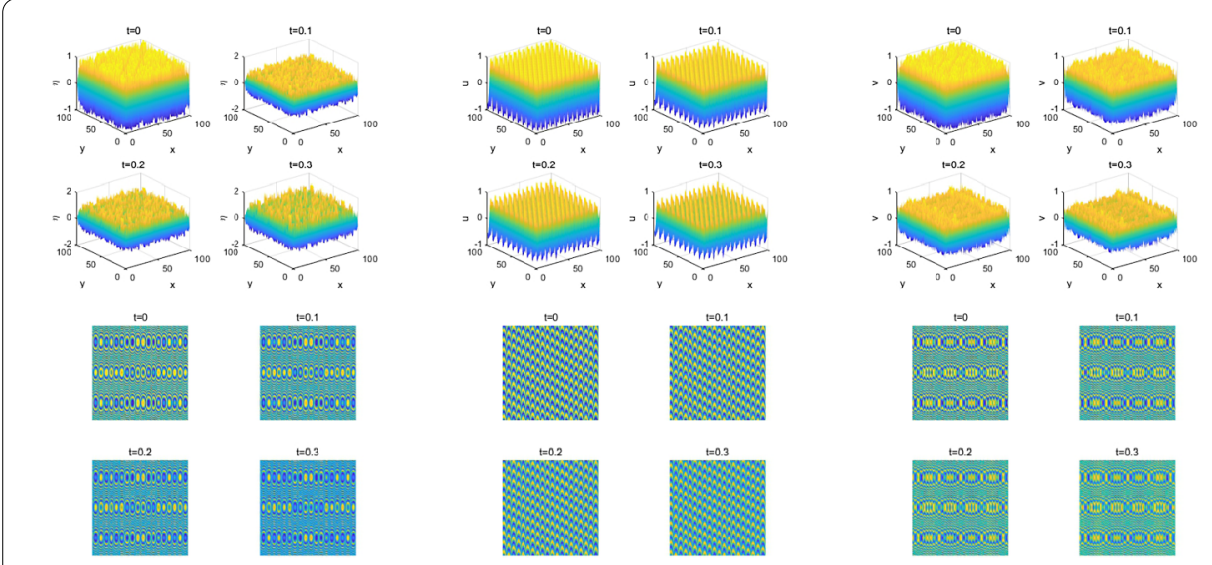

Figure 10 Numerical solution and pattern of Experiment 3, for initial conditions see Table 3

\section{Conclusions}

In this paper, a sinc function interpolation method has been, for the first time, built for a class of three species PPS with complex dynamical behavior. Some new complex dynam- 


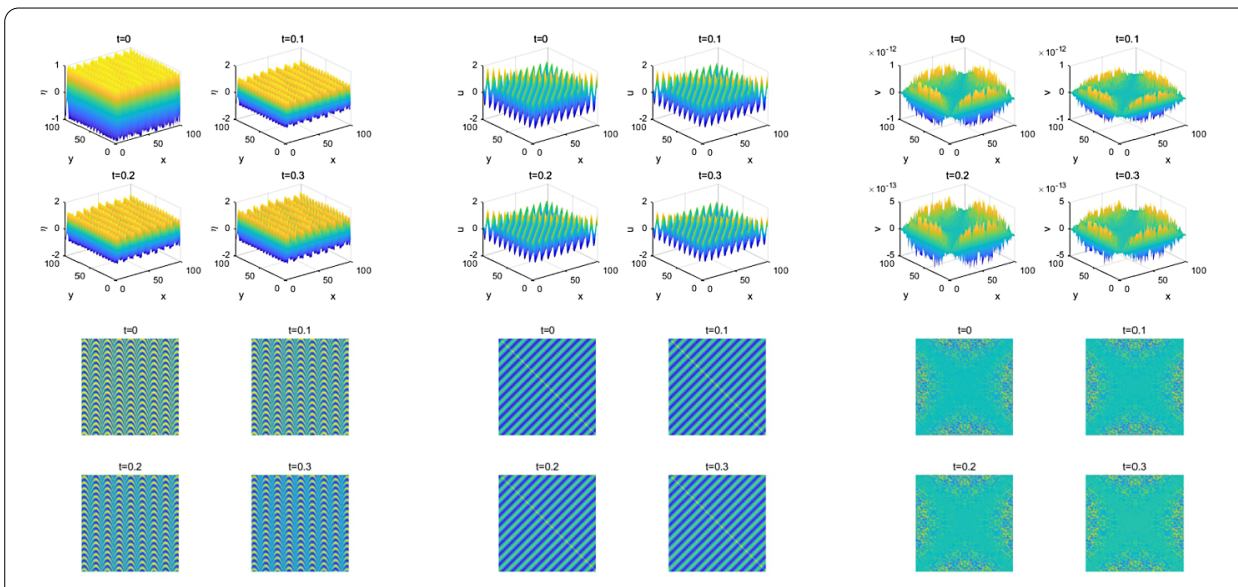

Figure 11 Numerical solution and pattern of Experiment 3, for initial conditions see Table 3

Table 1 Title of Figs. 1-11

\begin{tabular}{ll}
\hline Figure & Title \\
\hline Figs. 1-2 & Time series plots for Experiment 1 with different parameters, for parameters see Table 2 \\
Figs. 3-4 & Phase diagram of Experiment 1 with different parameters, for parameters see Table 2 \\
Figs. 5-7 & $\begin{array}{l}\text { Numerical solution and pattern of Experiment } 2 \text { with different initial conditions, for initial } \\
\text { conditions see Table } 3\end{array}$ \\
Figs. 8-11 & $\begin{array}{l}\text { Numerical solution and pattern of Experiment } 3 \text { with different initial conditions, for initial } \\
\text { conditions see Table } 3\end{array}$ \\
\hline
\end{tabular}

Table 2 Parameters of Figs. 1-11

\begin{tabular}{ll}
\hline Figure & Parameters \\
\hline Fig. 1 ( $(a)$ & $r=0.1, \alpha_{0}=0.6, \alpha_{1}=0.3, \beta_{0}=0.3, \beta_{1}=0.1, \gamma_{0}=0.1, \gamma_{1}=0.08, m_{1}=0.15, m_{2}=0.2, k=20$ \\
Fig. 1 (b) & $r=0.1, \alpha_{0}=0.6, \alpha_{1}=0.3, \beta_{0}=0.3, \beta_{1}=0.1, \gamma_{0}=0.1, \gamma_{1}=0.08, m_{1}=0.15, m_{2}=0.2, k=200$ \\
Fig. 1 (c) & $r=0.1, \alpha_{0}=0.6, \alpha_{1}=0.3, \beta_{0}=0.3, \beta_{1}=0.1, \gamma_{0}=0.1, \gamma_{1}=0.1, m_{1}=0.08, m_{2}=0.1, k=50$ \\
Fig. 2 ( $(a), 3$ & $r=0.6, \alpha_{0}=0.6, \alpha_{1}=0.3, \beta_{0}=0, \beta_{1}=0, \gamma_{0}=0.1, \gamma_{1}=0.1, m_{1}=0.08, m_{2}=0.1, k=200$ \\
Fig. 2 (b) & $r=0.6, \alpha_{0}=0.6, \alpha_{1}=0.3, \beta_{0}=0, \beta_{1}=0, \gamma_{0}=0.1, \gamma_{1}=0.1, m_{1}=0.08, m_{2}=0.1, k=50$ \\
Fig. 2 (c), 4 & $r=0.5, \alpha_{0}=0.3, \alpha_{1}=0.25, \beta_{0}=0, \beta_{1}=0, \gamma_{0}=0.2, \gamma_{1}=0.1, m_{1}=0.1, m_{2}=0.15, k=200$ \\
Figs. 5-7 & $r=0.1, \alpha_{0}=0.6, \alpha_{1}=0.3, \beta_{0}=0.3, \beta_{1}=0.1, \gamma_{0}=0.1, \gamma_{1}=1, m_{1}=0.3, m_{2}=0.5, k=1$ \\
Figs. 8-11 & $r=0.8, \alpha_{0}=0.4, \alpha_{1}=0.4, \beta_{0}=0.5, \beta_{1}=0.4, \gamma_{0}=0.2, \gamma_{1}=0.5, m_{1}=0.1, m_{2}=0.2, k=2.4$ \\
\hline
\end{tabular}

Table 3 Numerical solution and pattern of Experiments 2-3 with different initial condition of Figs. 5-11

\begin{tabular}{llll}
\hline Figure & $\eta(x, y, 0)$ & $u(x, y, 0)$ & $v(x, y, 0)$ \\
\hline Fig. 5 & $\operatorname{sech}\left(\sin \left(y x^{2}\right)\right)$ & $\operatorname{sech}\left(50 x^{2}+200 y-9\right)+\operatorname{ones}(N)$ & $\frac{4}{5} \sin \left(\cos \left(y^{2}-x^{2}\right)\right)$ \\
Fig. 6 & $\sin \left(\operatorname{sech}\left(\frac{x^{2}}{2}-y^{2}\right)\right)+\frac{1}{2}$ & $\sin \left(\cos \left(\frac{x^{2}}{2}+y^{2}\right)\right)+\frac{1}{2}$ & $-\sin \left(x^{2}+\frac{y^{2}}{10}\right)$ \\
Fig. 7 & $\operatorname{sech}\left(\frac{x}{2}+y^{3}\right)$ & $\cos \left(x^{2}+y^{2}\right)$ & $\sin \left(50 x^{2}+200 y-9\right)$ \\
Fig. 8 & $-\frac{1}{10} \sin \left(\frac{x^{2}+y^{2}}{10}\right)$ & $\cos \left(e^{-\sin \left(x^{2}+y^{2}\right)}\right)$ & $\operatorname{sech}\left(\operatorname{sech}\left(x^{2}+y\right)\right)$ \\
Fig. 9 & $\frac{1}{10} \sin \left(\frac{y^{2}-x^{2}}{10}\right)$ & $\pi \sin \left(50 x^{2}-y^{2}\right)+\frac{3}{5} \operatorname{rand}(N)$ & $\cos \left(x+\frac{y^{2}}{10}\right)$ \\
Fig. 10 & $-\sin \left(10 x^{2}+\frac{y^{2}}{10}\right)$ & $-\sin \left(\pi\left(\left(x-\frac{2}{5}\right)^{3}+\left(y+\frac{2}{5}\right)^{2}\right)\right)$ & $\sin \left(-x^{2}+\frac{y^{2}}{10}\right)$ \\
Fig. 11 & $\sin \left(50 x^{2}+200 y-9\right)$ & $\cos \left(\pi\left(\left(x-\frac{2}{5}\right)^{2}+\left(y+\frac{2}{5}\right)^{2}\right)\right)+\operatorname{sech}\left(20\left(\left(x+\frac{2}{5}\right)^{2}+\left(y-\frac{2}{5}\right)^{2}\right)\right)$ & $\sin \left(\pi\left(-x^{2}+y^{2}\right)\right)$ \\
\hline
\end{tabular}

ical behaviors are shown by using the present method. Simulation results were given to show the effectiveness of the present method and this system. 


\section{Acknowledgements}

We would like to express our deep thanks to the anonymous reviewers whose valuable comments and suggestions helped us improve this article greatly.

\section{Funding}

The work is supported by the Natural Science Foundation of Inner Mongolia [2017MS0103] and the National Natural Science Foundation of China [11361037].

\section{Abbreviations}

PPS, predator-prey system; BICM, barycentric interpolation collocation method; RKM, reproducing kernel method; VIM, variational iteration method; Ode45, Runge-Kutta method for ordinary differential equations.

\section{Availability of data and materials}

Data sharing not applicable to this article as no datasets were generated or analysed during the current study.

\section{Competing interests}

The authors declare that they have no competing interests.

\section{Authors' contributions}

All authors read and approved the final manuscript.

\section{Author details}

'Department of Mathematics, Inner Mongolia University of Technology, Hohhot, P.R. China. ${ }^{2}$ College of Physics, Jining Normal University, Jining, P.R. China. ${ }^{3}$ College of Statistics and Mathematics, Inner Mongolia University of Finance and Economics, Hohhot, P.R. China.

\section{Publisher's Note}

Springer Nature remains neutral with regard to jurisdictional claims in published maps and institutional affiliations.

Received: 29 December 2019 Accepted: 28 May 2020 Published online: 05 June 2020

\section{References}

1. Khajanchi, S.: Dynamic behavior of a Beddington-DeAngelis type stage structured predator-prey model. Appl. Math. Comput. 244, 344-360 (2014)

2. Khajanchi, S.: Modeling the dynamics of stage-structure predator-prey system with Monod-Haldane type response function. Appl. Math. Comput. 302, 122-143 (2017)

3. Banshidhar, S., Poria, S.: Dynamics of predator-prey system with fading memory. Appl. Math. Comput. 347, 319-333 (2019)

4. Gakkhar, S., Singh, B.: Dynamics of modified Leslie-Gower-type prey-predator model with seasonally varying parameters. Chaos Solitons Fractals 27, 1239-1255 (2006)

5. Paul, P., Ghosh, B., Kar, T.K.: Impact of species enrichment and fishing mortality in three species food chain models, Commun. Nonlinear Sci. Numer. Simul. 29, 208-223 (2015)

6. Tardieu, L., Roussel, S., Thompson, J.D., Labarraque, D., Salles, J.M.: Combining direct and indirect impacts to assess ecosystem service loss due to infrastructure construction. J. Environ. Manag. 152, 145-157 (2015)

7. Costa, R.M.S., Pavone, P.: Invasive plants and natural habitats: the role of alien species in the urban vegetation. Acta Hortic. 1215, 57-60 (2018)

8. Ghorai, S., Poria, S.: Pattern formation and control of spatiotemporal chaos in a reaction diffusion prey-predator system supplying additional food. Chaos Solitons Fractals 85, 57-67 (2016)

9. Liu, Z.J., Zhong, S.M., Yin, C., Chen, W.F.: Dynamics of impulsive reaction-diffusion predator-prey system with Holling type III functional response. Appl. Math. Model. 35, 5564-5578 (2011)

10. Pao, C.V: Global attractors of some predator-prey reaction-diffusion systems with density-dependent diffusion and time-delays. J. Math. Anal. Appl. 464, 164-187 (2018)

11. Eftimie, R.: Comment on "Towards a unified approach in the modeling of fibrosis: a review with research perspectives" by Martine Ben Amar and Carlo Bianca". Phys. Life Rev. 17, 90-91 (2016)

12. Alzahrani, T., Eftimie, R., Trucu, D.: Multiscale moving boundary modelling of cancer interactions with a fusogenic oncolytic virus: the impact of syncytia dynamics. Math. Biosci. (2019). https://doi.org/10.1016/..mbs.2019.108296

13. Alzahrani, T., Eftimie, R., Trucu, D.: Multiscale modelling of cancer response to oncolytic viral therapy. Math. Biosci. 310, 76-95 (2019)

14. Mishra, P., Raw, S.N., Tiwari, B.: Study of a Leslie-Gower predator-prey model with prey defense and mutual interference of predators. Chaos Solitons Fractals 120, 1-16 (2019)

15. Mbroh, N.A., Munyakazi, J.B.: A fitted operator finite difference method of lines for singularly perturbed parabolic convection-diffusion problems. Math. Comput. Simul. 165, 156-171 (2019)

16. Dhiman, N., Tamsir, M.: A collocation technique based on modified form of trigonometric cubic B-spline basis functions for Fisher's reaction-diffusion equation. Multidiscip. Model. Mater. Struct. 14, 923-939 (2018)

17. Elliott, C.M., Ranner, T.: Evolving surface finite element method for the Cahn-Hilliard equation. Numer. Math. 129, 483-534 (2015)

18. Garvie, M.R., Trenchea, C.: Finite element approximation of spatially extended predator-prey interactions with the Holling type II functional response. Numer. Math. 107, 641-667 (2007)

19. Wu, H., Han, X.F.: Discontinuous Galerkin spectral element method for a class of nonlinear reaction-diffusion equations. J. Shanghai Univ. Nat. Sci. 20, 768 (2014) 
20. Rashid, A., Ismail, A.I.Md.: A Fourier pseudospectral method for solving coupled viscous Burgers' equations. Comput. Methods Appl. Math. 9, 412-420 (2009)

21. Dehghan, M., Abbaszadeh, M.: Variational multiscale element free Galerkin and local discontinuous Galerkin methods for solving two-dimensional Brusselator reaction-diffusion system with and without cross-diffusion. Comput. Methods Appl. Mech. Eng. 300, 770-797 (2016)

22. He, J.H.: Homotopy perturbation method: a new nonlinear analytical technique. Appl. Math. Comput. 135, 73-79 (2003)

23. He, J.-H., Wu, X.-H.: Construction of solitary solution and compacton-like solution by variational iteration method. Chaos Solitons Fractals 29, 108-113 (2006)

24. Du, M.J., Li, J.M., Wang, Y.L., Zhang, W.: Numerical simulation of a class of three-dimensional Kolmogorov model with chaotic dynamic behavior by using barycentric interpolation collocation method. Complexity 2019, Article ID 3426974 (2019)

25. Liu, F.F., Wang, Y.L., Li, S.G.: Barycentric interpolation collocation method for solving the coupled viscous Burgers equations. Int. J. Comput. Math. 95, 2162-2173 (2018)

26. Du, M.J., Wang, Y.L.: Some novel complex dynamic behaviors of a class of four-dimensional chaotic or hyperchaotic system based on a meshless collocation method. Complexity 2019, Article ID 5034025 (2019)

27. Zhou, X.F., Li, J.M., Wang, Y.L., Zhang, W.: Numerical simulation of a class of hyperchaotic system using barycentric Lagrange interpolation collocation method. Complexity 2019, Article ID 1739785 (2019)

28. Wang, Y.L., Temuer, C.L., Pang, J.: New algorithm for second-order boundary value problems of integro-differential equation. J. Comput. Appl. Math. 229, 1-6 (2009)

29. Wang, Y.L., Du, M.J., Tan, F.G.: Using reproducing kernel for solving a class of fractional partial differential equation with non-classical conditions. Appl. Math. Comput. 219, 5918-5925 (2013)

30. Wang, Y.L., Su, L.J., Cao, X.J.: Using reproducing kernel for solving a class of singularly perturbed problems. Comput. Math. Appl. 61, 421-430 (2011)

31. Wang, Y.L., Temuer, C.L.: Using reproducing kernel for solving a class of singular weakly nonlinear boundary value problems. Int. J. Comput. Math. 87, 367-380 (2010)

32. Wang, Y.L., Li, Z.Y: A new method for solving a class of mixed boundary value problems with singular coefficient. Appl. Math. Comput. 217, 2768-2772 (2010)

33. Zhang, X.: Efficient Solution of Differential Equation Based on MATLAB: Spectral Method Principle and Implementation. Mechanical Industry Press, Beijing (2015)

\section{Submit your manuscript to a SpringerOpen ${ }^{\circ}$ journal and benefit from:}

- Convenient online submission

- Rigorous peer review

- Open access: articles freely available online

- High visibility within the field

- Retaining the copyright to your article

Submit your next manuscript at $\boldsymbol{~ s p r i n g e r o p e n . c o m ~}$ 